\title{
XI. On a new reaction between electrostatic tubes and insulators, and on the electrostatic field round an electric current, and the theory of Professor Poynting
}

\section{M.W. de Nicolaiève}

To cite this article: M.W. de Nicolaiève (1902) XI. On a new reaction between electrostatic tubes and insulators, and on the electrostatic field round an electric current, and the theory of Professor Poynting, Philosophical Magazine Series 6, 4:19, 133-138, DOI: 10.1080/14786440209462824

To link to this article: http://dx.doi.org/10.1080/14786440209462824

\section{Published online: 15 Apr 2009.}

Submit your article to this journal ¿

\section{Article views: 2}




\section{[ 133 ]}

XI. On a New Reaction between Electrostatic Tulies and Insulators, and on the Electroseatic Field round an Electric Current, and the Theory of Professor Poynting. By M. W. DE NiColaiève*.

$\mathrm{O}^{N \text { a new reaction letween electrostatic tules and insulators.- }}$ This special reaction is observed in the electrostatic field which the author has shown to exist in electrolysis during the passage of the current. The tubes of force of this field coincide with the lines of current, and so insulating matter which is dielectric for tubes of the ordinary field behaves in an electrolyte like a perfect dia-electric deprived of electric permeability.

First Experiment.-Place in distilled water a system of two vertical plates perpendicular to a third, $A B$, fig. 1 , all insulating, the horizontal section having an $\mathrm{H}$-form. In the compartments formed by this plate immerse two strips of tin 15 to $20 \mathrm{cms}$. long with horizontal sections represented by $K$ and $L$. When the strips are connected up to a source of current they diverge under the influ-

Fig, 1.

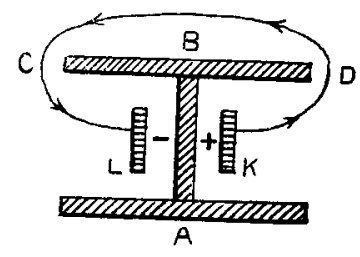
ence of tubes of the kind represented by $\mathrm{K} \mathrm{D} \mathrm{B} \mathrm{C} \mathrm{L,} \mathrm{as} \mathrm{repre-}$ sented in elevation in fig. 2 . If they are connected up to the

Fig. 2.

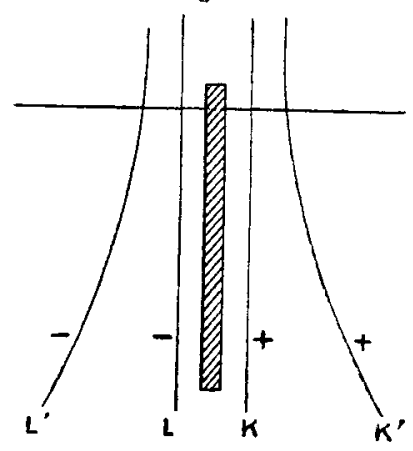

terminals of a 300 -volt transformer their extremities are displaced as much as $20 \mathrm{mms}$. outwards. The set of tubes like $\mathrm{K}$ D B C L can only exist through the action of the diaphragm

* From the Comptes Rendus, 30th December, 1901, and 6th Jaruary, 1902, with some additions by the Author. Communicated by Prof. J. H. Poynting. 
upon them, and these tubes will have a reaction tending to compress the diaphragm.

Second Experiment-Two strips $\mathrm{K}$ and L (fig. 3) are suspended in water and separated from each other by a glass partition, A B. The electrostatic tubes of the kind $\mathrm{L} A \mathrm{~K}$ displace the strips into the positions $\mathrm{K}^{\prime}$ and $\mathrm{L}^{\prime}$, so that the two bodies under their mutual actions are displaced in the same direction, which is contrary to the fundamental law of Action and Reaction. The only platasible explanation is that the edge $A$ reacts upon the tubes $L A K$ which act like stretched elastic threads pressing against the edge and pulling the strips.

Fig. 3.

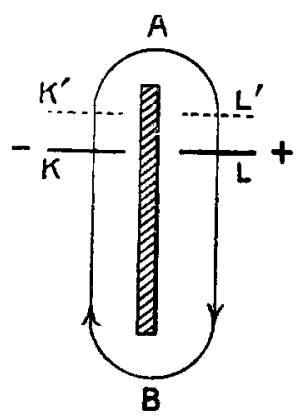

Third Experiment.-Metallic wires or strips $\mathrm{K}$ and $\mathrm{L}$ (fig. 4) are immersed in water and are fixed. The mica

Fig 4.

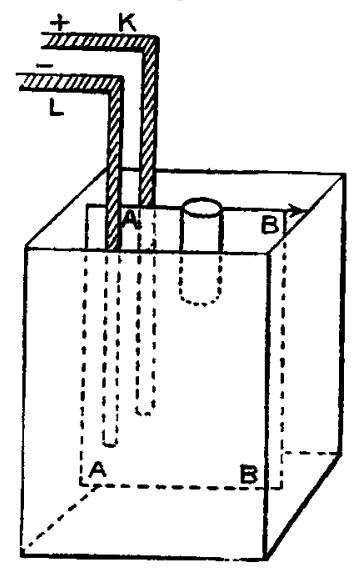

partition, A B, floated by a cork, is movable. When in the position $A B$ it moves from $A$ towards $B$, in the opposite direction to the movement of the strips in the second experiment. The moving force is the difference of the pulls of the tubes L A K and L B K (fig. 3). When the edge A has passed the line of the electrodes K $L$ the tubes issuing from the other faces of the electrodes act still in the same direction. When the mica is parallel to the plane through K L it retreats from them, a movement which is produced by the pressures 
of the tubes. Such pressures should be experienced by the walls of vessels containing electrolytes. To confirm the experiment and to be assured that the movements are not due to currents of liquid, two electrolytes were placed in series, both consisting of distilled water. After the mica had moved in one, a small quantity of chloride of sodium was dissolved in it. The current became stronger, but the electrostatic field became weaker and the mica showed hardly any trace of movement. Tinfoil floating in the water and replacing the mica behaved in the opposite way. The author intends to make the third experiment in rarefied gases, where by ionization the electrostatic tubes may also coincide with the tubes of current, but where the influence of the statical lines upon the walls will also come into account.

Fourth Experiment. - A mica pendulum (fig. 5) movable round a light axis of glass is placed between the terminals of a Holtz machine with the axis parallel to the line joining the

Fig 5 .

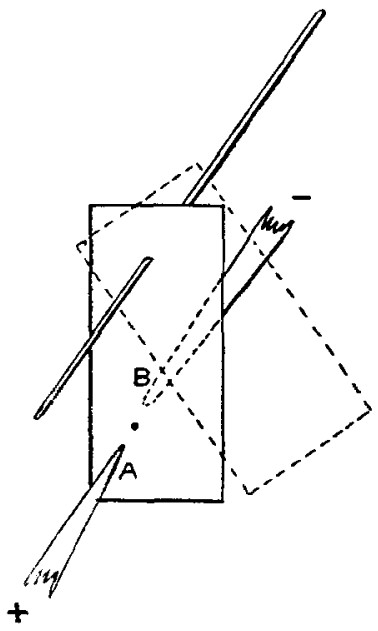

Fig. 6.
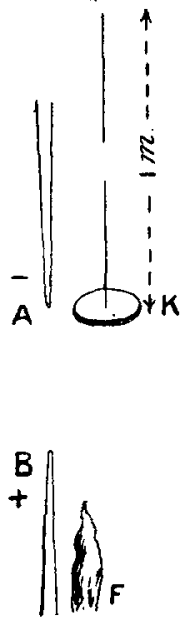

terminals. If this line passes through the plate and the spark is obliged to turn from the most direct route, the pendulum retreats from the terminals. If the air is ionized by the discharges the effect may be attributed to the reactions of the electrostatic tubes on the edge of the mica, but if not, we cannot so explain the motion. The two faces of the mica may perhaps be electrified by charges of the same sign respectively as those in the points $A$ and $B$, and the displacement to the right may be due to repulsion of like charges. It would be necessary to put a flame under the points and 
the mica to take away the charges from the latter and to facilitate the ionization of the air, but the illness of the author has interrupted this experiment. He has made the following experiment. A disk of mica $\mathrm{K}$ (fig. 6) is suspended excentrically to one side of the line of two-pointed terminals connected to a Holtz machine. When the flame $\mathrm{F}$ is lighted the disk $\mathrm{K}$ is displaced to the riglit by the current of hot air. But when the electric machine is in action, the disk is displaced at least twice as much.

\section{On the Electrostatic Field round an Electric Current.}

Apparatus.-In a large glass tube placed rertically are suspended two loops, A E ( and B F D (fig. 7), parallel to each other, 4 to $5 \mathrm{~mm}$. apart, and consisting of two strips of tinfoil,

Fig. 7.

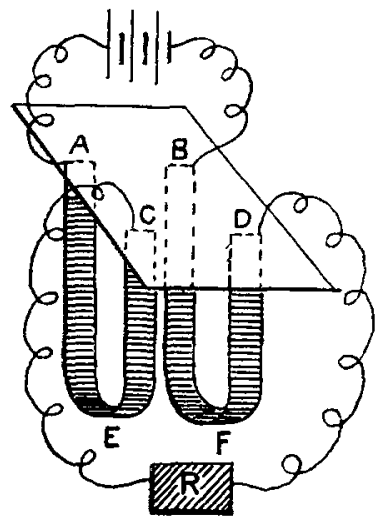

$3 \mathrm{~mm}$. wide, hanging down to a depth of $50 \mathrm{~cm}$. The greater this depth the more marked is the effect. The free ends, A B CD, are fixed in a card placed on the top of the tube.

The points $A$ and $B$ of the loops are connected up to the terminals of a battery of 100 volts. The points $\mathrm{C}$ and $\mathrm{D}$ are either insulated or are connected through a rheostat of glowlamps. In the first case the loops are charged to the potentials of the battery terminals; in the second case a constant current flows through the two loops in opposite directions so that the electromagnetic forces tend to make them repel each other.

First Experiment.-The battery is unconnected, and the loops are at rest. Then the battery is joined up and the locis move towards each other. The attraction is produced by the system of electrostatic tubes from the one loop to the other.

Second Experiment.-The battery circuit is completed by 
a rheostat of 900 ohms. The movement towards each other persists and differs little from that on open circuit. The: repulsive electromagnetic action exists, but does not manifest itself owing to the feebleness of the current $(0.11 \mathrm{amp}$.$) .$ There is only one probable explanation. The electrostatic tubes join the two loops in the same way as in the statical case, and the mechanical effect remains nearly the same, because on the one hand through the small fall of potential the tubes have nearly the same strength, and on the other the electromagnetic repulsion is very small.

In the statical condition the difference of potential between the two ends of each tube is the same and the system of tubes is in equilibrium. In the dynamical condition the differences of potential diminish from 100 volts to zero, and the equilibrium of the tubes is disturbed. The transversal pressures diminish gradually, and the tubes are displaced perpendicularly to their axes, sliding along the conductors and sweeping through the dielectric medium. The fall of potential along the circuit shows that each tube, after an infinitely small displacement, will have a less difference of potential between its ends, an effect which may be set lown to the destructive discharge of the end-cellules in the body of the conductors. We see that the experiment leads to results exactly agreeing with the theory of Professor Poynting, and that it confirms that theory. When the inserted resistance is diminished to $200 \mathrm{ohms}$ the attraction changes to electromagnetic repulsion. As the electrostatic action is inversely proportional to the squares of the distances, and the electromagnetic action is inversely proportional to the distances, we can make either prevail by varying the distance.

Third Experiment.-In naphtha the attraction increases.

Fourth Experiment.-Cutting out the rheostat and immersing the loops in distilled water the attraction becomes very powerful and is manifested at a distance of 4 or $5 \mathrm{~cm}$. To get rid of the small liquid currents, and to be sure of the electric character of the attraction, the author put on an alternating current from a transformer and observed the attraction while he dissolved larger and larger quantities of some salt : the current and the electrolysis went on gradually increasing, but the difference of potential between the loops decreased, and at the same time the attraction decreased down to zero. In this experiment the electrostatic tubes are propagated in the water transversely to the conducting loop; they are destroyed in the liquid itself by means of an intermolecular discharge (by the aid of ions), and the lines of current are directed along the electrostatic tubes. As the tensions of these tubes are, before destruction, several times 
greater than in air, a more powerful attraction is observed than in air.

Fifth Experiment.-Two vertical strips of tinfoil, parallel to each other 4 or $5 \mathrm{~mm}$. apart, are immersed in water, a plate of glass or of mica a little wider than the strips is interposed, and while the current is on, a repulsion of the strips is observed. The electrostatic tubes coincide with the lines of electrolytic current, and as the plate deviates the tubes uniting the interior and opposite faces, the resultant of the attractions becomes smaller than that of the repulsions due to the tubes applied to the exterior faces. To direct the Faraday tubes so as to give preponderance to those which pull the strips apart, it is necessary to arrange as in fig. 8 , that is, to fix to the diaphragm two perpendicular plates which do not allow the tubes (and lines of current) to scatter laterally; the divergence of the leaves is thus increased several fold.

Sixth Experiment.-Two vertical strips of tinfoil are immersed in water (fig. 9). They are parallel, and as near each other as is possible consistent with not being drawn together by capillarity. The pair of strips constitutes one electrode, while the other is a vertical metallic wire, placed in the plane of the first electrode, and $3 \mathrm{~cm}$. distant from it. On the passage of the current, the strips move a little towards the wire, and at the same time diverge from each other just as if they were in air before an electrified conductor.

The 4th, 5th, and 6th experiments show the existence of the electrostatic field inside an electrolyte.

Seventh Experiment.-If the two linear conductors in Herz's experiment are replaced by two flexible loops 15 or $20 \mathrm{~cm}$. apart, they attract each other

Fig. 8.

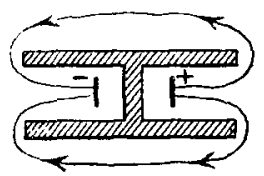
powerfully. Here the tubes are in motion between the linear conductors along which they slide by their extremities, and this is the cause of the equality of velocity of propagation in the medium and in conductors.

Physical Laboratory of the Academy of Sciences, St. Petersburg. 